\title{
Rheagogies: Modelling non-trophic effects in food webs
}

\author{
Roger Arditi ${ }^{\mathrm{a}, *}$, Jerzy Michalski ${ }^{\mathrm{b}, 1}$, Alexandre H. Hirzel ${ }^{\mathrm{c}}$ \\ ${ }^{a}$ Ecologie des Populations et Communautés, Institut National Agronomique Paris-Grignon, 16 rue Claude Bernard, \\ 75231 Paris Cedex 05, France \\ ${ }^{\mathrm{b}}$ Department of Mathematics, Swiss Federal Institute of Technology, 1015 Lausanne, Switzerland \\ ${ }^{\mathrm{c}}$ Institute of Zoology and Animal Ecology, University of Lausanne, 1015 Lausanne, Switzerland
}

Received 4 October 2004; received in revised form 5 April 2005; accepted 11 April 2005

Available online 4 June 2005

\begin{abstract}
In a food web, nutrients flow via trophic links. For this reason, trophic interactions have a fundamental character due to the principle of mass conservation. To further comply with this principle, we consider a food web model that includes nutrient cycling. Non-trophic effects amongst species of the food web are modelled as interaction modifications, i.e., a functional change in the trophic interaction between two species caused by a third species (a three-party interaction that we call "rheagogy"). We also consider that the ecological communities modelled by the food webs result from an assembly process that involves colonisations and extinctions. We find that two distinct classes of ecological communities must be distinguished: (a) "superefficient" communities, in which almost all available nutrients are incorporated into the biomass and (b) "sub-efficient" ones, in which a large proportion of nutrients is not fixed by living organisms. We show that rheagogies (that model non-trophic interactions) are crucial: the larger the effects of rheagogies, the easier the construction of super-efficient communities. These communities are characterized by positive rheagogies, meaning that a certain proportion of mutualistic interactions is necessary. Systems with few or weak rheagogies are less likely to use available abiotic resources efficiently. Although richness (i.e., number of species) is also positively related to efficient nutrient use, its importance is much smaller than that of rheagogies.

(C) 2005 Elsevier B.V. All rights reserved.
\end{abstract}

Keywords: Interaction modifications; Ecosystem engineers; Community assembly; Nutrient cycling

\section{Introduction}

Much theoretical and field effort has been devoted to study food web structures and food web dynamics (see, e.g., Drossel and McKane (2003), Berlow et al. (2004)

\footnotetext{
* Corresponding author.

E-mail address: arditi@inapg.fr (R. Arditi).

${ }^{1}$ Present address: Chemin de la Possession 12, 1066 Epalinges, Switzerland
}

for recent reviews; Pimm $(1982,1991)$ for reviews of older work). In a food web, nutrients flow via trophic links. For this reason, trophic interactions (e.g., herbivory, predation, exploitation competition) have a basic, direct, character due to the fundamental principle of mass conservation. This can explain why other types of ecological interactions (particularly interference competition and mutualism) have often been ignored in food web studies (as emphasized by Berlow et al., 2004). To further comply with the principle of mass 
conservation, many food web models include nutrient cycling. Since the total quantity of nutrients is necessarily limited in such closed systems, this prevents populations from growing to infinity. This approach also allows to study the links between structural properties (e.g., diversity) and functional properties (e.g., primary production) (e.g., DeAngelis, 1992; Grover, 1994; Loreau, 1994; Neutel et al., 1994).

Organisms of particular interest that are actors of non-trophic interactions are the ecosystem engineers. Lawton and Jones (1995) introduced this term to designate organisms that "directly or indirectly modulate availability of resources (other than themselves) to other species, by causing physical state changes in biotic or abiotic materials". Beavers are a paradigmatic example of such engineers. They create ponds, which have profound effects on resource availability to many other species. Other examples include earthworms, trees, coral reefs, planktonic organisms or big mammals (see Jones et al., 1997 for a review). One way to formalise the effect of ecosystem engineers is to consider them as "interaction modifiers". We define an interaction modification as a change in the direct interaction between two species due to the density of a third species (Wootton, 1993; see also Billick and Case, 1994 for discussion). For example, the mere presence of a carnivorous predator can modify the foraging efficiency of one of its prey, a predator can affect prey behaviour making it more vulnerable to another predator, inedible phytoplankton can reduce the grazing efficiency of zooplankton, a sessile species can provide a refuge for prey and change in this way the searching efficiency of its predator.

Actually, in the food web approach in which binary interactions are trophic links only, all other types of interactions (including mutualism, interference competition, amensalism, commensalism and the effects of engineers) must be considered as modifications of the trophic interactions. As mentioned above, this is necessary in order to conform to mass conservation. For example, two predator species that interfere with each other decrease each other's efficiency to exploit their respective prey. Mutualists increase each other's capacity to feed or to avoid their respective predators.

To formalise interaction modifications in food web models, we define a rheagogy (from the Greek rheô, flow and agôge $\hat{o}$, influence), as the situation in which a change in the density of one species alters the trophic
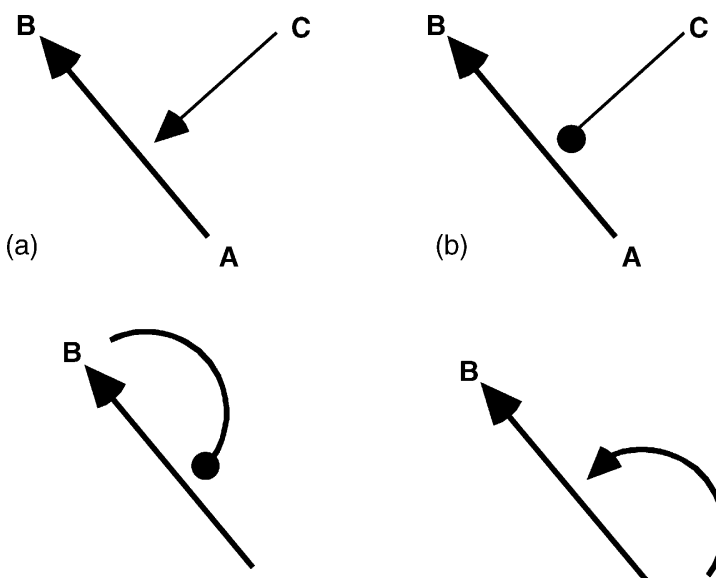

(c)

A

(d)

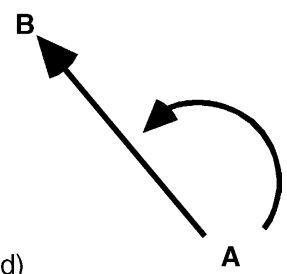

Fig. 1. A rheagogy is a formalisation of non-trophic influences in food webs. Species B eats species A, i.e., nutrients flow from A to B. Species $C$ (that must belong to the food web) influences this flow either (a) by reinforcing it or (b) by weakening it. Special cases are those of species exerting rheagogies on their own trophic interactions (e.g., (c and d)); this can model density-dependent predation.

interaction (i.e., the flow of matter) between two other species (Fig. 1). In general, the modifiers do not have to take part in the flows between the trophically connected organisms they affect (Fig. 1a and b). Of course, in order to subsist, modifiers must consume some resource; thus, they must belong to the food web. Special cases of rheagogies involving trophically connected species can model density-dependent predation (Fig. 1c and d).

A common theoretical approach to ecological communities is to view them as resulting from processes of assembly and succession. Colonisers invade successfully or fail, resident species can go extinct and the species composition of the system changes before a final community settles down (e.g., Post and Pimm, 1983; Drake, 1990; Luh and Pimm, 1993; Morton et al., 1996; see Pimm, 1991 and Law, 1999 for reviews). A model situation is that of a newly emerged island that is colonised from a nearby continent, considered as a species pool. Assembly and succession are clearly at work in any system affected by a strong perturbation: a grassland after a drought, a forest after a fire or an attack of bark beetles, a lake after a strong pollution.

In this paper, we will consider communities that are built by species assembly and that include nutrient 
cycling. Our purpose is to study the effect of rheagogies (as defined above, as interaction modifications). More precisely, we will examine the following questions:

- How do rheagogies affect community construction?

- How do rheagogies affect the structure of assembled communities?

- How does the complexity of the species pool affect the stability and functioning of the assembled community?

\section{Food web structure}

\subsection{A trophic structure with nutrient cycling}

Our food web model contains four trophic levels: plants, herbivores, carnivores and super-carnivores. Species from each trophic level can only feed on the immediately inferior trophic level (no omnivory, no intraguild predation, no cannibalism). Consumers do not assimilate all nutrients extracted from resources. Non-assimilated nutrients return to the soil as detritus or excrements and become available to the plants. All plants feed on the same, unique, nutrient compartment (soil).

It would be possible to include one more compartment, through which all detritus would pass before being decomposed into mineral nutrients available to plants. For the sake of simplicity, we assume that this mineralisation is sufficiently fast that the detritus can be assumed to be converted instantaneously into nutrients.

We base our model on the well-known Generalised Lotka-Volterra (GLV) equations:

$\frac{\mathrm{d} X_{i}}{\mathrm{~d} t}=X_{i}\left(r_{i}+\sum_{j=1}^{m} \gamma_{i j} X_{j}\right)$,

where $X_{i}$ is the abundance of species $i$ (in this paper abundance is measured as the amount of nutrients in the biomass of species $(i), r_{i}$ the intrinsic growth rate and $\gamma_{i j}$ is the influence of species $j$ on the growth rate of species $i$ and $m$ is the number of species in the system.

In its most general definition, the GLV model does not impose any particular structure to the coefficients $\gamma_{i j}$ : a priori, $\gamma_{i j}$ and $\gamma_{j i}$ are independent. The case in which both are negative is usually interpreted as competition, the case in which both are positive, as mutualism. However, since we want to comply with the principle of mass conservation and keep track of nutrient cycling, we cannot use the GLV model in this wide sense. We must restrict it to the case of trophic links, i.e., the case in which $\gamma_{i j}$ and $\gamma_{j i}$ have opposite signs. However, this Lotka-Volterra model for predator-prey interactions is known to suffer serious theoretical defects, particularly because it assumes a linear functional response without saturation and without predator dependence (Arditi and Ginzburg, 1989). Several theoretical studies of food web models resting on more complex interaction terms have been undertaken in the past decade (Berryman et al., 1995; Michalski and Arditi, 1995a,b; Arditi and Michalski, 1996; McCann et al., 1998; Pelletier, 2000; Drossel et al., 2001, 2004; Quince et al., 2002). Nevertheless, it can be shown that the GLV model is a reasonable approximation of almost any general food web model provided that this model has a non-trivial equilibrium (see Appendix B in Michalski and Arditi (1999) for a mathematical derivation). This is necessarily the case of the present model because it is a closed system with a fixed total amount of nutrients.

Making explicit our special trophic structure gives the following system:

Soil:

$$
\begin{aligned}
\frac{\mathrm{d} X_{0}}{\mathrm{~d} t}= & \sum_{i=1}^{m} X_{i}\left(b_{i}+(1-e) \sum_{j \in \operatorname{res}(i)} a_{j i} X_{j}\right) \\
& -X_{0} \sum_{i \in \text { plants }} a_{0 i} X_{i}
\end{aligned}
$$

Plants:

$\frac{\mathrm{d} X_{i}}{\mathrm{~d} t}=X_{i}\left(-b_{i}+a_{0 i} X_{0}-\sum_{j \in \operatorname{cons}(i)} a_{i j} X_{j}\right)$,

Animals (three levels):

$\frac{\mathrm{d} X_{i}}{\mathrm{~d} t}=X_{i}\left(-b_{i}+e \sum_{k \in \operatorname{res}(i)} a_{k i} X_{k}-\sum_{j \in \operatorname{cons}(i)} a_{i j} X_{j}\right)$,

where "res( $(i)$ " stands for the set of resources of species $i$, "cons $(i)$ " stands for the set of consumers of species $i$, and $e$ is the conversion efficiency of consumers, assumed the same for all species, $e<1$. In (2), 
in order to make clear the meaning of each term, we assumed that all parameters $b_{i}$ and $a_{i j}$ have positive values. Note that no species can increase without a resource: in isolation, each species decreases at rate $b_{i}$.

Note that plants only can use nutrients from the soil $X_{0}: a_{0 i}=0$ if species $i$ is not a plant. Animals can consume plants or other animals from the inferior trophic level. The sum of all right-hand sides of (2) is zero, i.e., the total amount of nutrients in the system does not vary, reflecting our assumption that the system is closed.

The principle of mass conservation imposes a strict structure on the parameters $a_{i j}$ : the interactions described by them must be of the predator-prey type. Note that, if $i$ is a prey and $j$ its predator, only parameter $a_{i j}$ is used; the parameter $a_{j i}$ is not used and needs not be defined. Consumed prey biomass that is not converted into predator biomass (with efficiency $e$ ) returns to the soil (in proportion $1-e$ ). By restricting the model to trophic interactions only, we make sure that there are no nutrients that appear out of nothing or that disappear.

\subsection{Plant growth}

Note that the plant equations (2b) has the same form as that for animals (2c), while in the ecological literature plant growth is frequently described by the logistic equation. In fact, plant logistic growth is included implicitly in (2). To see this more clearly, consider a system composed of one plant only with abundance $X_{1}$ and described by (2):

Soil:

$\frac{\mathrm{d} X_{0}}{\mathrm{~d} t}=b_{1} X_{1}-X_{0} a_{01} X_{1}$,

Plant:

$\frac{\mathrm{d} X_{1}}{\mathrm{~d} t}=X_{1}\left(-b_{1}+a_{01} X_{0}\right)$.

As the sum of the right hand sides of (3) is zero, the total quantity of nutrients, is constant: $X_{0}+X_{1}=Q=$ constant. Substituting $X_{0}=Q-X_{1}$ into (3b) gives

$\frac{\mathrm{d} X_{1}}{\mathrm{~d} t}=X_{1}\left(a_{01} Q-b_{1}-a_{01} X_{1}\right)$ which is the logistic equation $\mathrm{d} X_{1} / \mathrm{d} t=r X_{1}(1-$ $\left.X_{1} / K\right)$ with intrinsic growth rate

$r=a_{01} Q-b_{1}$

and carrying capacity

$K=Q-\frac{b_{1}}{a_{01}}$.

Therefore, logistic growth can be understood as a consequence of nutrient cycling. In our model, nutrients are explicitly recycled and Equations (2) can be considered as an extension of logistic growth of plants in a food web with many species and several trophic levels.

\subsection{Rheagogies}

With the parameters $a_{i j}$ constant, equations (2) describe a system with purely trophic interactions. Rheagogies can be included into (2) if we allow the parameters $a_{i j}$ to be functions of species abundances. This can be done by replacing the interaction terms of model (2) by:

$a_{i j} \max \left(0, a_{i j}\left[1+\sum_{k=1}^{m} c_{i j k} X_{k}\right]\right)$

where $c_{i j k}$ describes the influence of species $k$ on the trophic interaction between the resource $i$ and the consumer $j,(i, j, k=1,2, \ldots, m)$. Species $k$ needs not be involved in a direct trophic interaction with species $i$ and $j$, but it may be so. It may even be identical to $i$ or $j$. Parameters $c_{i j k}$ can be positive or negative but the expression (6) is bounded from below by 0 . This is necessary in order to forbid the reversal of the nutrient flow between species $i$ and $j$ : whatever the influence of other species, a hare will never eat a lynx.

In this model, different rheagogies on a given trophic link are considered as additive. This is a crude approximation of what takes place in reality, where interaction modifications can interfere with each other. However, this approximation is in line with the general simplification of the functioning of the ecological community. Putting more realism into the model for rheagogies would require putting more realism into the model for trophic interactions and would lead us far beyond the simple Generalised Lotka-Volterra equations (1). In this study, we have chosen to work 
with a simple exploratory model that can give results that are easy to understand and to interpret. The rheagogy model (6) is on the same level of approximation as the trophic model (1).

The equations (2) together with the modification (6) fully describe the food web model with its trophic structure (four trophic levels, no omnivory, no intraguild predation, no cannibalism), the nutrient compartment (soil), nutrient recycling and rheagogies.

\section{Food web assembly}

We examine here an assembly and succession process, in which a new community is constructed via invasions from a species pool. In such a situation, the invading populations arrive at very low densities, much lower than those of the resident populations. A new invader must find resources in order to grow; for example, the first species to succeed an invasion must be a plant; thereafter, a herbivore eating this plant may succeed, and so on. When a new coloniser arrives, one of the following events can happen:

- it fails (no resources, competition too hard) and is rejected;

- it is simply added to the system, modifying the equilibrium densities of other species;

- it replaces another, less competitive species;

- its arrival disturbs the system in such way that multiple extinctions follow; this avalanche of extinctions may include the new coloniser.

We assume that species from the species pool arrive rather frequently to the new community. This assumption is in contrast with many simulation studies of assembly dynamics found in the literature. Usually, after each invasion, authors leave sufficient time for the system to reach a new equilibrium before a new invasion is allowed (Robinson and Valentine, 1979; Post and Pimm, 1983; Taylor, 1988; Drake, 1990; Case, 1991; Grover, 1994). This was done mainly for historical reasons: some 25 years ago, when food web assembly started to be explored by means of numerical simulation, computers were not fast enough to calculate, in reasonable time, the whole temporal dynamics. Instead, equilibria were calculated directly (which is an easy computational task with the
Generalised Lotka-Volterra Equations (1)) and sets of "assembly rules" (not always biologically or mathematically justified; see Morton et al., 1996) were applied. As we now have fast computers, we will no longer follow the unrealistic assumption that invaders wait until the system reaches an equilibrium.

Another argument against "waiting till equilibrium" is that it only allows the construction of very small communities when nutrients are recycled. In systems with nutrient cycling, like those described by (2), the whole community stands on one nutrient reservoir only. [Systems with many coupled nutrient reservoirs can also be considered (see, e.g., Huston and DeAngelis, 1994 and Loreau, 1996) but the discussion of this subject goes beyond the scope of our simple exploratory model.] Grover (1994) analysed assembly sequences of systems of food chains, each chain composed of one plant and its herbivore, all chains being based on the same nutrient reservoir. He found that, in order to construct a large system, invading species must arrive in a strictly determined sequence if one waits till equilibrium before allowing a next invasion. In particular, if one wants to construct a stable system composed of two plants and their respective herbivores (each herbivore eating only one plant) then, out of 24 possible invasion sequences (a species being given only one possibility to invade) only one leads to the complete system with four species; half of them end up with only one plant present and only three of them give systems with two plants and one herbivore (Grover, 1994).

In our case, the system is more complex than that studied by Grover (1994): we have four trophic levels, we have generalists, and species can try to invade several times. When we checked our simulation program with the rule of "waiting till equilibrium", we obtained qualitatively the same results as those of Grover (1994): the probability to obtain a large system via community assembly with infrequent invasions is very low in the presence of nutrient recycling and with only one nutrient reservoir. Our simulations typically presented the following scheme: a plant colonised the new system and reached its equilibrium abundance; then a herbivore arrived and the system of two species reached a new equilibrium; after a possible arrival of a carnivore and, possibly, a super-carnivore, a second plant arrived 


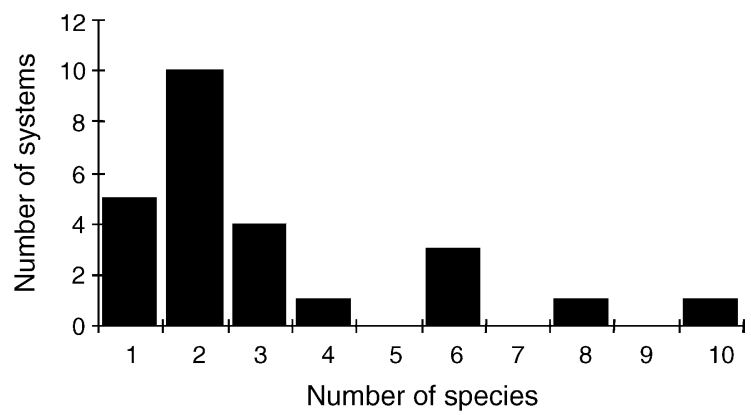

Fig. 2. Distribution of the richness of assembled communities, obtained in simulations where a new colonisation is allowed only after the system has reached an equilibrium. $S=100, C_{t}=0.19$, $c=1$ and $C_{r}=0.3$. Sample size: 25 simulations.

sooner or later. If it was more competitive than the first one (weakened by its herbivore), it eliminated the first one and the whole food chain with it. This process continued until the end of the simulation, giving a final system with, on average, three species (Fig. 2; see below for simulation details). For the reasons explained earlier, our subsequent simulations will use frequent invasions instead.

In some early theoretical studies of food web assembly, systems with many species could be obtained despite infrequent invasions (Robinson and Valentine, 1979; Post and Pimm, 1983; Taylor, 1988; Drake, 1990; Case, 1991). However, this was possible only because, in these studies, each plant species had its own separate resource (i.e., separate carrying capacities).

\section{Simulation details}

\subsection{The algorithm}

(1) The species pool is created. It consists of a "potential" food web of four trophic levels with equal numbers of species on each level, without omnivory, and characterised by the parameters $b_{i}$, $a_{i j}$ and $c_{i j k}$, sampled randomly in a uniform distribution over appropriate intervals (see next section). The components of complexity in the species pool, i.e., the species richness, the number of trophic links and the number of rheagogies can all be modified.
(2) A species, chosen randomly from the species pool, invades. The choice of this species must fulfil two conditions in the assembled community:

(a) it is not already present;

(b) it has a resource (prey).

In order to preserve the total quantity of nutrients in the system, the initial biomass of the invader (0.01) is subtracted from the soil.

(3) The temporal evolution of the assembled system is calculated by integrating the equations (2) and (6).

(4) Every species whose abundance drops below the threshold (0.005) is eliminated. Its biomass is returned to the soil. A new, smaller system is thus created.

(5) During the first 200 time units, there is a probability $P=0.1$ that a new species from the species pool colonises the system after each time unit (i.e., there is one colonisation every 10 time units, on average).

(6) After 200 time units, invasions are interrupted and the system is left to evolve until it reaches an equilibrium, or the limit of 5000 time units is reached.

(7) Repeat steps (2)-(6) for different random colonisation sequences.

\subsection{Parameter choice}

We first run simulations for assembly dynamics with equations (2) alone (i.e., without the rheagogies (6)) in order to choose parameter values in such way that

- stable systems with four trophic levels could be obtained;

- roughly half of the total nutrient content would be in the biomass.

The first requirement is obvious: we want to study food webs of non-trivial complexity. The reason for the second requirement is that one of our aims is to study the influence of rheagogies on community functioning: Will their presence increase or decrease the efficiency with which the ecological community uses available nutrients? 
We found that these two requirements were fulfilled with the following parameter choice. The parameter values are taken from uniform distributions over the following intervals:

$b_{i} \in[4,5]$ for plants, then divided by 11 at each upper trophic level (simple allometric rule);

$a_{i i}=0$ (no cannibalism);

$a_{i j}=0$ if $i$ and $j$ do not belong to consecutive trophic levels (no omnivory, no intra-guild predation);

otherwise

$a_{i j}=0$ with probability $1-C_{t}$;

$a_{i j} \in[0.001,0.01]$ with probability $C_{t}$;

$e=0.3$ is the conversion efficiency of consumers;

$Q=1000$ is the total quantity of nutrients in the system;

$X_{\text {init }}=0.01$ is the initial abundance of colonisers.

The richness of the species pool, $S$, could be 40,60 , $80,100,120$ or 140 . The proportion of trophic interactions, $C_{t}$, is the standard definition of connectance (thereafter called the "trophic connectance" to be distinguished from the rheagogic connectance defined later). In our simulations, $C_{t}$ could take the values $0.04,0.08,0.11,0.15,0.19,0.23,0.27,0.30$, 0.34 and 0.38 or, respectively, $10 \%, 20 \%, 30 \%, 40 \%$, $50 \%, 60 \%, 70 \%, 80 \%, 90 \%$ and $100 \%$ of all possible interactions of a food web with four trophic levels, with equal numbers of species on each level and without omnivory.

Note that, with the most favourable choice for a plant species, i.e., $b_{i}=4$ and $a_{0 i}=0.01$, the carrying capacity of the plant species (if alone in the system) is, according to (5b), equal to 600 . This value lies roughly in the middle between 0 and 1000 , the total quantity of nutrients in the system.

The parameters dealing with rheagogies are chosen in the following way:

If $a_{i j}=0$ then $c_{i j k}=0(i, j, k=1,2, \ldots, m)$

otherwise

$c_{i j k} \in[-c, c]$ with probability $C_{r}$ and

$c_{i j k}=0$ with probability $1-C_{r}$.

The maximal rheagogic intensity, $c$, could vary between 0 and 1.4. The probability $C_{r}$ is called the "rheagogic connectance" in analogy with $C_{t}$. The rheagogic connectance could take all values between 0 and 1 , with a step of 0.1 .

\section{Results}

The model developed in the previous sections was used by varying systematically the three community parameters of the species pool (richness $S$, trophic connectance $C_{t}$, rheagogic connectance $C_{r}$ ). In the assembled community, these descriptors were left free to evolve as a consequence of colonisations and extinctions.

A clear segregation process appears: the total quantity of available nutrients being set to 1000 , the biomass of assembled systems is either less than 600 or higher than 900 (Fig. 3). This allows to distinguish two classes of communities:

sub-efficient systems (average biomass 32.4\%), in which a large part of the nutrients remains in the soil; super-efficient systems, in which $90 \%$ or more of the nutrients are concentrated in the biomass (average $99.7 \%$ ), with almost no nutrients remaining in the soil.

The rheagogic parameters $c_{i j k}$ were chosen in the interval $[-c, c]$. Fig. 4 shows that, the higher the maximal rheagogic intensity $c$, the higher the proportion of super-efficient systems. In other words, a stronger influence of modifiers on the trophic interactions increases the probability that the assembly process will produce a super-efficient system.

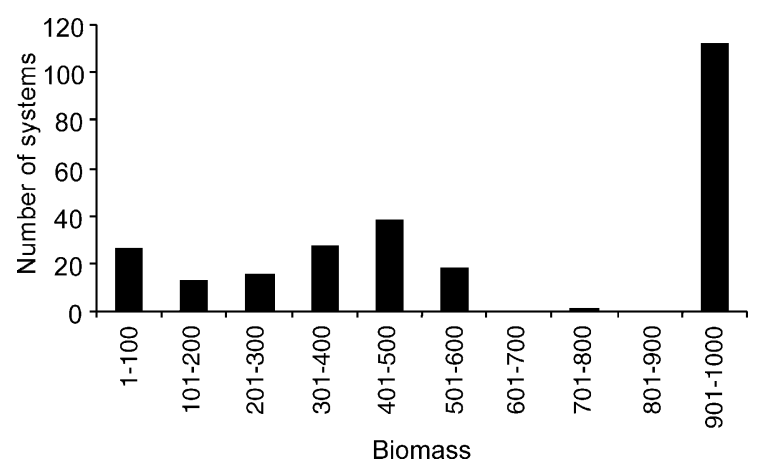

Fig. 3. Distribution of biomasses of assembled communities. The histogram represents 250 simulation runs covering all values of trophic connectances $C_{t} . S=100, c=1$ and $C_{r}=0.5$. 


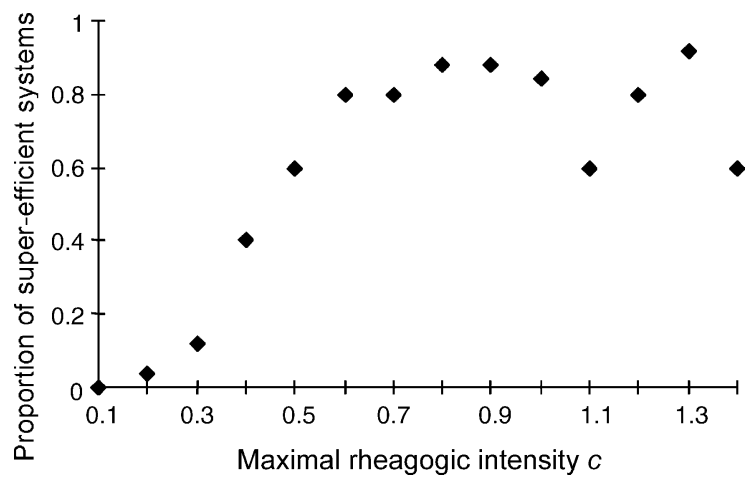

Fig. 4. Proportion of super-efficient systems as a function of the maximal rheagogic intensity $c . S=100, C_{t}=0.19$ and $C_{r}=0.5$. Each point is the mean of 25 simulations.

Setting $c=0.4$, super-efficiency was studied in response to combined variations of all three parameters of the species pool: $S, C_{t}$ and $C_{r}$ Since there are no correlations among these three parameters, their effect on super-efficiency can be detected by simple independent correlations. Fig. 5c shows that superefficiency is strongly favoured by the rheagogic connectance $C_{r}(r=0.785, P<0.001)$. The trophic connectance acts in the opposite direction: Fig. $5 b$ shows that the probability to obtain a super-efficient community is negatively correlated with $C_{t}$; this correlation is weak but significant $(r=-0.151$, $P<0.001)$. The richness $S$ has no significant influence (Fig. 5a, $r=0.045$, NS).

Comparing the two classes of assembled communities, we notice that, besides their differences of biomass (Fig. 3), they also exhibit very distinct differences in their mean rheagogic intensity and in their richness. With $S=100, C_{t}$ varying between 0.04 and $0.38, c=1, C_{r}$ varying between 0 and 1 , the mean realised rheagogic intensity is positive in super-efficient systems $\left(\left\langle c_{i j k}\right\rangle=0.034\right.$, S.D. $=0.145, n=1013, P<$ $0.001)$ and negative in sub-efficient systems $\left(\left\langle c_{i j k}\right\rangle=\right.$ -0.189 , S.D. $=0.328, n=362, P<0.001)$, each with wide variability. The realised richness of super-efficient systems $(12.89 \pm 6.82)$ is, on average, five times higher than that of sub-efficient ones $(2.30 \pm 1.57)$. This suggests that more diverse assembled communities are also more likely to be super-efficient. To verify this, we kept track of the biomasses and the number of species of all systems constructed from a given species pool with $S=100 . \quad C_{t}=0.19, \quad c=1, C_{r}=0.5$. Each of 100 simulation runs differed from others only by the
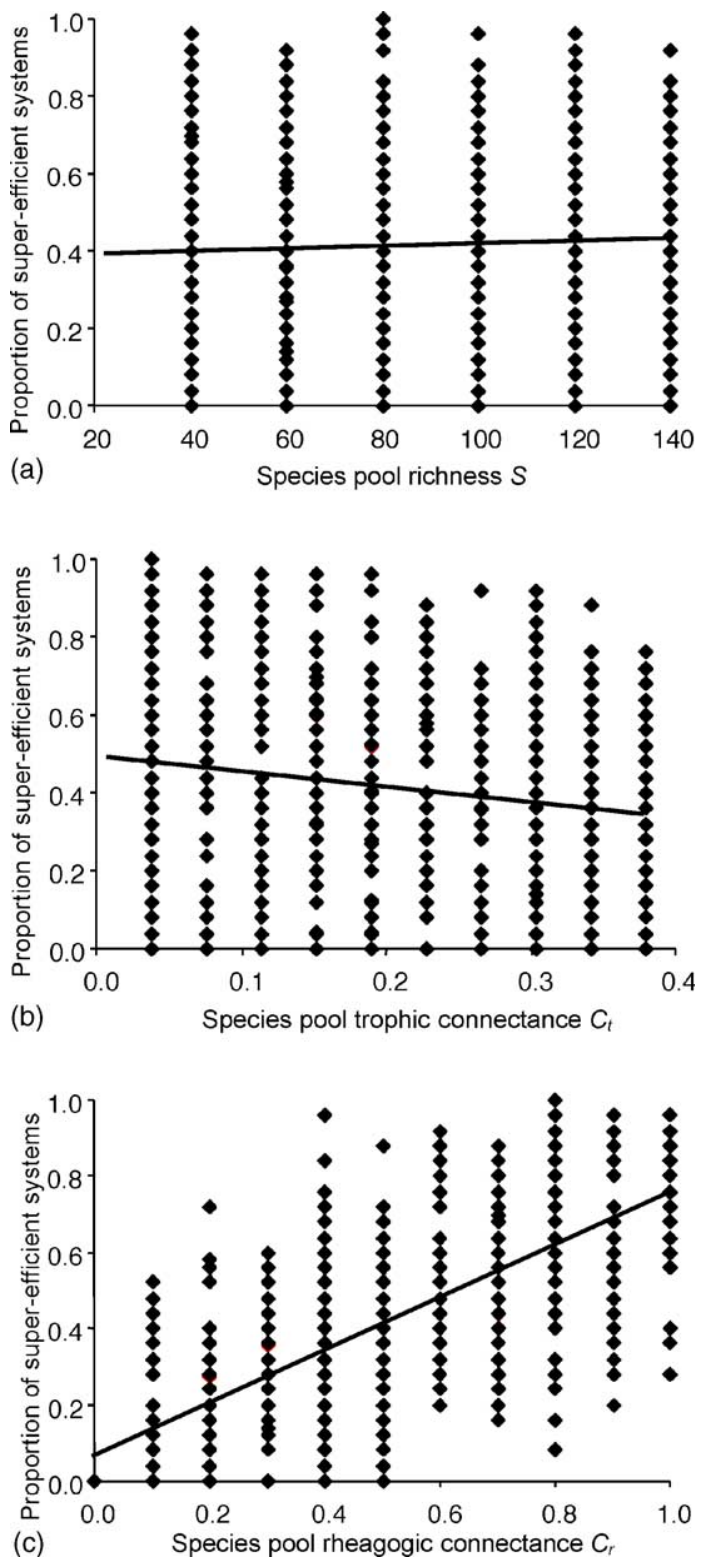

Fig. 5. Proportion of super-efficient systems as a function of the three parameters of the species pool: (a) richness $S$, (b) trophic connectance $C_{t}$, (c) rheagogic connectance $C_{r}$. In each graph, $S$ varies between 40 and 140, $C_{t}$ varies between 0.04 and $0.38, C_{r}$ varies between 0 and $1, c=0.4$. Each point is the mean of 25 simulations with the same parameter values.

sequence in which colonisers arrived. The richness of the resulting communities varied from 0 (no species at all) to 28. As expected, Fig. 6 shows that all assembled communities richer than 10 were super-efficient. Note 


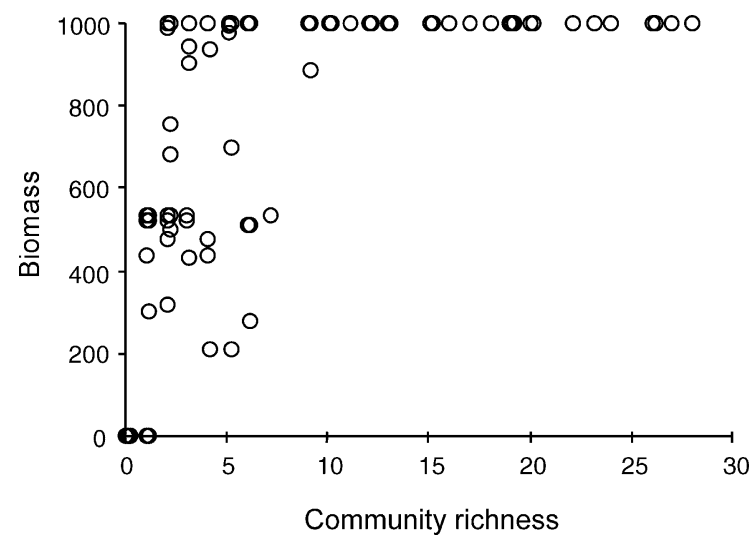

Fig. 6. The biomass of the assembled communities as a function of the number of species in the same communities. Results from 100 simulation runs with one species pool with parameters $S=100$, $C_{t}=0.19, c=1$ and $C_{r}=0.5$.

that poorer communities could be either sub-efficient or super-efficient.

\section{Discussion}

In summary, we have built a dynamic model with nutrient cycling that is meant to study the importance of non-trophic effects in food webs. Non-trophic interactions are represented as modifiers of the trophic interactions-more precisely as rheagogies, a class of interaction modifications in which a third species modifies the trophic interaction between two species. We have considered ecological communities formed in a process of assembly and succession, i.e., communities constructed by colonisations from a species pool. We have found that two very distinct classes of ecological communities can be distinguished: (a) "super-efficient" ones, which incorporate almost all available nutrients and (b) "sub-efficient" ones, in which a large part of nutrients is left in the "soil".

The probability that the final community be superefficient is positively correlated with the two parameters that characterise rheagogies: the rheagogic connectance $C_{r}$ and the maximal rheagogic intensity $c$. In other words, these two factors promote the productivity of ecosystems. Moreover, super-efficient communities are characterized by positive rheagogies, meaning that a certain proportion of mutualistic interactions is necessary.
Without rheagogies (i.e., when $c=0$ or $C_{r}=0$ ), super-efficient systems cannot be constructed (Figs. 4 and $5 \mathrm{c}$ ). With our choice of parameter values, no "super-plant" was able to use all available nutrients without help (via rheagogies) of other species. This means that, if trophic interactions are the only interactions among the species forming the community, then the available nutrients cannot be used efficiently, i.e., nutrients cannot be the limiting factor.

The richness of the species pool has almost no influence: a three-fold increase of the species pool (from 40 to 120) makes the fraction of super-efficient systems increase by only about $7 \%$ (see Fig. 5a). However, super-efficient assembled communities are much more species-rich than sub-efficient ones (Fig. 6). Thus, the more species there are in a realised community, the more chance there is that it is superefficient. This also means that the efficiency of nutrient use is positively correlated with the realised richness.

Field experiments that study the relations between species richness and ecosystem functioning suggest that ecosystem productivity is positively correlated with biodiversity (Naeem et al., 1996; Tilman et al., 1996) and that limiting resources are more completely utilised in places with greater diversity of species (Tilman et al., 1996; Hooper and Vitousek, 1997). Although these experiments provided convincing demonstrations of the effects of biodiversity on productivity and soil nutrient concentration, the underlying mechanisms for these effects are not clear. Our simulation studies are not directly comparable with these experiments: we considered assembled communities in equilibrium while the experiments considered ecosystem productivities during one season. Nevertheless, our results provide one possible explanation for the observed correlation between richness and efficient nutrient use. We have demonstrated that more rheagogies and more species are, by themselves, sufficient to explain qualitatively the observed correlation (Figs. 4, 5c and 6).

In real ecological communities, all species must have trophic interactions but non-trophic interactions are also numerous. In such complex natural systems, it is not clear, a priori, what is the net effect of nontrophic interactions. Modelling these as rheagogies, our simulation results suggest that non-trophic interactions (particularly facilitations) are crucial: the process of assembly and succession tends to select 
highly effective communities; the larger the effects of rheagogies, the easier this selection.

\section{Acknowledgements}

We thank Michel Loreau for stimulating discussions in the early phase of the work. This work was supported by the Swiss National Science Foundation and by various French CNRS grants. It is a contribution of the French CoReV network.

\section{References}

Arditi, R., Ginzburg, L.R., 1989. Coupling in predator-prey dynamics: ratio dependence. J. Theor. Biol. 139, 311-326.

Arditi, R., Michalski, J., 1996. Nonlinear food web models and their responses to increased basal productivity. In: Polis, G.A., Winemiller, K.O. (Eds.), Food Webs: Integration of Patterns and Dynamics. Chapman and Hall, New York, pp. 122-133.

Berlow, E.L., et al., 2004. Interaction strengths in food webs: issues and opportunities. J. Anim. Ecol. 73, 585-598.

Berryman, A.A., Michalski, J., Gutierrez, A.P., Arditi, R., 1995. Logistic theory of food web dynamics. Ecology 76, 336-343.

Billick, I., Case, T.J., 1994. Higher order interactions in ecological communities: what are they and how can they be detected? Ecology 75, 1529-1543.

Case, T.J., 1991. Invasion resistance, species build-up and community collapse in metapopulation models with interspecies competition. Biol. J. Linn. Soc. 42, 239-266.

DeAngelis, D.L., 1992. Dynamics of Nutrient Cycling and Food Webs. Chapman and Hall, London.

Drake, J.A., 1990. The mechanics of community assembly and succession. J. Theor. Biol. 147, 213-233.

Drossel, B., McKane, A.J., 2003. Modelling food webs. In: Bornholdt, S., Schuster, H.G. (Eds.), Handbook of Graphs and Networks: From the Genome to the Internet. Wiley-VCH, Berlin.

Drossel, B., Higgs, P.G., McKane, A.J., 2001. The influence of predator-prey population dynamics on the long-term evolution of food web structure. J. Theor. Biol. 208, 91-107.

Drossel, B., McKane, A.J., Quince, C., 2004. The impact of nonlinear functional responses on the long-term evolution of food web structure. J. Theor. Biol. 229, 539-548.

Grover, J.P., 1994. Assembly rules for communities of nutrientlimited plants and specialist herbivores. Am. Nat. 143, 258-282.

Hooper, D.U., Vitousek, P.M., 1997. The effects of plant composition and diversity on ecosystem processes. Science $277,1302-1305$.

Huston, M.A., DeAngelis, D.L., 1994. Competition and coexistence: the effects of resource transport and supply rates. Am. Nat. 144, 954-977.

Jones, C.G., Lawton, J.H., Shachak, M., 1997. Positive and negative effects of organisms as physical ecosystem engineers. Ecology 78, 1946-1957.
Law, R., 1999. Theoretical aspects of community assembly. In: McGlade, J. (Ed.), Advanced Ecological Theory: Principles and Applications. Blackwell, pp. 143-171.

Lawton, J.H., Jones, C.G., 1995. Linking species and ecosystems: organisms as ecosystem engineers. In: Jones, C.G., Lawton, J.H. (Eds.), Linking Species and Ecosystems. Chapman and Hall, New York, pp. 141-150.

Loreau, M., 1994. Material cycling and the stability of ecosystems. Am. Nat. 143, 508-513.

Loreau, M., 1996. Coexistence of multiple food chains in a heterogeneous environment: interactions among community structure, ecosystem functioning, and nutrient dynamics. Math. Biosci. 134, 153-188.

Luh, H.K., Pimm, S.L., 1993. The assembly of ecological communities: a minimalist approach. J. Anim. Ecol. 62, 749-765.

McCann, K., Hastings, A., Huxel, G.R., 1998. Weak trophic interactions and the balance of nature. Nature 395, 794-798.

Michalski, J., Arditi, R., 1995a. Food webs with predator interference. J. Biol. Syst. 3, 323-330.

Michalski, J., Arditi, R., 1995b. Food web structure at equilibrium and far from it: is it the same? Proc. R. Soc. Lond. B 259, $217-$ 222.

Michalski, J., Arditi, R., 1999. The complexity-stability problem in food web theory. What can we learn from exploratory models? In: Blasco, F., Weill, A. (Eds.), Advances in Environmental and Ecological Modelling. Elsevier, Paris, pp. 91-119.

Morton, R.D., Law, R., Pimm, S.L., Drake, J.A., 1996. On models for assembling ecological communities. Oikos 75, 493-499.

Naeem, S., Hakansson, K., Lawton, J.H., Crawley, M.J., Thompson, L.J., 1996. Biodiversity and plant productivity in a model assemblage of plant species. Oikos 76, 259-264.

Neutel, A.M., Roerdink, J.B.T.M., de Ruiter, P.C., 1994. Global stability of two-level detritus decomposer food chains. J. Theor. Biol. 171, 351-353.

Pelletier, J.D., 2000. Are large complex ecosystems more unstable? A theoretical reassessment with predator switching. Math. Biosci. 163, 91-96.

Pimm, S.L., 1982. Food Webs. Chapman and Hall, London.

Pimm, S.L., 1991. Balance of Nature? The University of Chicago Press, Chicago.

Post, W.M., Pimm, S.L., 1983. Community assembly and food web stability. Math. Biosci. 64, 169-192.

Quince, C., Higgs, P.G., McKane, A.J., 2002. Food web structure and the evolution of ecological communities. In: Lässig, M., Valleriani, A. (Eds.), Lecture Notes in Physics, vol. 585. Springer, Berlin, pp. 281-298.

Robinson, J.V., Valentine, W.D., 1979. The concepts of elasticity, invulnerability and invadability. J. Theor. Biol. 81, 91-104.

Taylor, P.J., 1988. The construction and turnover of complex community models having Generalized Lotka-Volterra dynamics. J. Theor. Biol. 135, 569-588.

Tilman, D., Wedin, D., Knops, J., 1996. Productivity and sustainability influenced by biodiversity in grassland ecosystems. Nature 379, 718-720.

Wootton, J.T., 1993. Indirect effects and habitat use in an intertidal community: interaction chains and interaction modifications. Am. Nat. 141, 71-89. 